\title{
DOSAGE FORM DEVELOPMENT AND PRELIMINARY PHYSICOCHEMICAL CHARACTERIZATION OF TRIKANTAKADI KVATHA
}

\section{SWATI SHARMA, DILEEP SINGH BAGHEL*, SAURABH SINGH, SACHIN KUMAR SINGH}

Department of Ayurvedic Pharmacy, School of Pharmaceutical Sciences, Lovely Professional University, Phagwara, Punjab, India. Email: baghel_12@rediffmail.com

Received: 14 July 2017, Revised and Accepted: 25 July 2017

\section{ABSTRACT}

Objective: This is aimed to study the development of different dosage form and physicochemical characterization of Trikantakadi Kvatha (TK).

Methods: Stability, shelf life, non-convenient, and large dosages administration are the major concern for Kvatha. To overcome these problems, an effort has been made to modify the formulation without changing its efficacy into various dosage forms such as tablet, syrup, and tincture. Comparative pharmacognostic, physicochemical, and phytochemical parameters of crude herbs and prepared formulations were investigated. TK was prepared by classical method mentioned in literature and converted into TK syrup, TK Ghana vati, and Trikantakadi tincture (TT). Precaution should be taken during the processing of formulations. TT placed at a dark place in airtight container.

Results: Physicochemical and phytochemical investigations are not shown any remarkable variations with various prepared dosage forms. The $\mathrm{R}_{\mathrm{f}}$ range observed between the 0.08 and 0.80 follows the standard value when compared with the reference of plant drug used for the preparation of dosage form.

Conclusion: The prepared dosages forms were not exhibited any remarkable difference according to thin-layer chromatography studies and physicochemical parameters. However, the developed dosage forms are more stable than kvatha.

Keyword: Trikantakadi kvatha, Ghana vati, Syrup, Tincture.

(C) 2017 The Authors. Published by Innovare Academic Sciences Pvt Ltd. This is an open access article under the CC BY license (http://creativecommons. org/licenses/by/4. 0/) DOI: http://dx.doi.org/10.22159/ajpcr.2017.v10s3.21336

\section{INTRODUCTION}

Kvatha one of the most popular Kalpana among five basic Kalpanas widely used therapeutically (to cure the different ailment) as well as pharmaceutically (used to prepare the different dosage forms like Ghana, vati, syrup, etc. Although a clear description is not available in Vedic literature, a detailed description is present in all Samhitas regarding its preparation, amount of water, and reduction in volume of liquid. Kvatha Kalpana may be defined as a Kalpana in which a specific quantity of Kvatha Dravya is taken and specific amount of water is used as menstruum and applying specific quantum of heat, the volume of water is reduced to specific amount and then by filtering Kvatha can be obtained [1].

Trikantakadi kvatha (TK) is an aqueous extract hence it is always questioned against its stability period. Hence, that modification in the dosage form was attempted in the present study and modified dosage forms such as TK syrup (TKS), Trikantakadi tincture (TT), and TK Ghana Vati (TKGV) were prepared. To ensure the stability period of prepared dosage form, the stability study was done and compared the observed value of all the physicochemical properties with the previous data.

\section{Chronicled appraisal}

Reference is cited for Kvatha Dravya along with nature and various proportion of water taken for the preparation of kvatha (aqueous extract) (Table 1a). In Ayurveda Sara Samgraha and Rasatantrasara Va Sidhaprayoga Samgraha mentioned the use of TK in Asmari, Mutrakricha, Mutraghat and to remove the kidney stones outside the body (Table 1b) [7,8].

\section{METHODS}

All the raw herbs are collected from the local market of Jalandhar and authentication is carried out by Dr. Satiwinderjeet Kaur, Head, Department of Botanical and Environmental Sciences, Guru Nanak
Dev University Amritsar, Punjab, with ref. no. 1088, dated 18.10.2016. Microscopic analysis of raw materials was studied according to the methods of the Quality Standard of Indian Medicinal Plant (Tables 2 - Figs. 1-6 and Table 3 - Figs. 7-12) [9].

\section{Method of preparation}

Preparation of TK

TK was prepared according to the procedure mentioned in the Ayurveda Sar Samgraha [7].

TKS

$500 \mathrm{~g}$ of sugar candy powder was added to previously prepared TK (1000 ml). Citric acid (0.1 g), propylparaben ( $2 \mathrm{~g})$, and methylparaben ( $2 \mathrm{~g}$ ) were used as a preservative.

\section{TKGV}

Granules were prepared from $80 \mathrm{~g}$ of TKG and mix $80 \mathrm{~g}$ of a mixture of herbal drugs (TK) powder for making the tablet $550 \mathrm{mg}$ by adding

Table 1a: Kvatha Dravya along with their types of water and proportion of water

\begin{tabular}{llll}
\hline Reference book & $\begin{array}{l}\text { Nature } \\
\text { of drugs/ } \\
\text { quantity of } \\
\text { drugs }\end{array}$ & $\begin{array}{l}\text { Quantity } \\
\text { of water }\end{array}$ & $\begin{array}{l}\text { Reduction } \\
\text { up to }\end{array}$ \\
& 4 tola (48 g) & 16 times & $1 / 8^{\text {th }}$ \\
\hline Charaka Samhita [2] & - & 16 times & $1 / 4^{\text {th }}$ \\
Sushruta Samhita [3] & - & 8 times & $1 / 4^{\text {th }}$ \\
Astanga Samgraha [4] & Madhyam & 16 times & $1 / 8^{\text {th }}$ \\
Sharangdhara Samhita [5] & - & 26 times & $1 / 8^{\text {th }}$ \\
Yogatarangini [6] & - & &
\end{tabular}


Table 1b: Literature review on TK

\begin{tabular}{|c|c|c|c|c|c|c|}
\hline Name of drugs & Common name & B. N. and family & Part used & A. S. S. & R. Va S. S. & Quantity \\
\hline Gokshura & Gokhru & $\begin{array}{l}\text { Tribulus terrestris Linn. } \\
\text { Zygophyllaceae }\end{array}$ & Fruit & + & + & 1 Part \\
\hline Amaltaas ka gudha (pulp) & Girimaal, Amaltaas & $\begin{array}{l}\text { Cassia fistula Linn. } \\
\text { Fabaceae }\end{array}$ & Fruit pulp & + & + & 1 Part \\
\hline Darbhmoola & Doob & $\begin{array}{l}\text { Cynodon dactylon Linn. } \\
\text { Poaceae }\end{array}$ & Root & + & + & 1 Part \\
\hline Damasha/javasha & Javasa & $\begin{array}{l}\text { Alhagi camelorum (Bieb). Desv. } \\
\text { Fabaceae }\end{array}$ & Whole part & + & + & 1 Part \\
\hline Pashan bheda & Silphara, Pakhanabheda & $\begin{array}{l}\text { Bergenia ligulata (Haw.) Sternb. } \\
\text { Saxifragaceae }\end{array}$ & Root & + & + & 1 Part \\
\hline Harar & Harad, Harre & $\begin{array}{l}\text { Terminalia chebula } \text { Retz. } \\
\text { Combretaceae }\end{array}$ & Fruit & + & + & 1 Part \\
\hline Kaasmool & Kasa, Kans & $\begin{array}{l}\text { Saccharum spontaneum Linn. } \\
\text { Poaceae }\end{array}$ & Root & + & + & 1 Part \\
\hline Pitpapda & Dhamgajra & $\begin{array}{l}\text { Fumaria vaillantii Lam. } \\
\text { Fumaraceae }\end{array}$ & Whole plant & + & - & 1 Part \\
\hline
\end{tabular}

Table 2: Microscopical characters of Raw materials

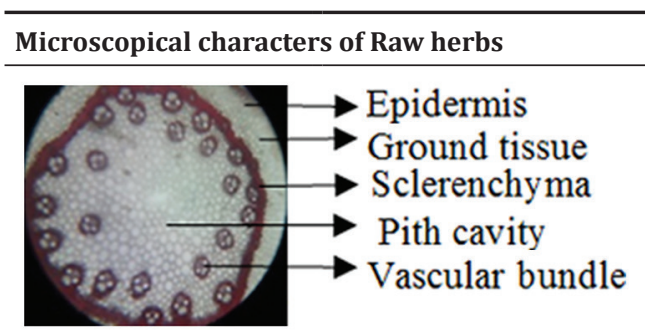

Fig. 1: Durbha (Root)

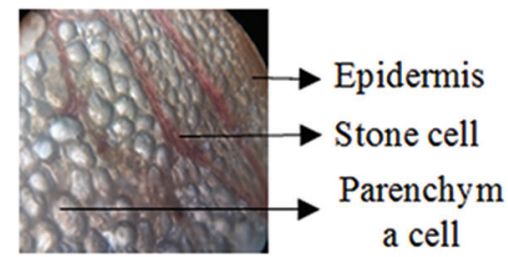

Fig. 4: Harar (Fruit pulp)

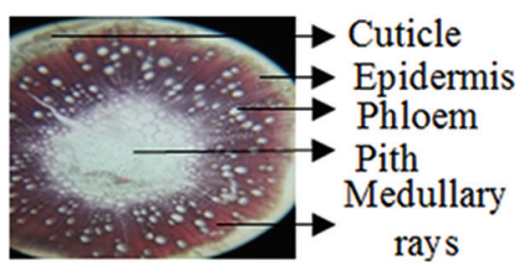

Fig. 2: Javasha (Stem part)

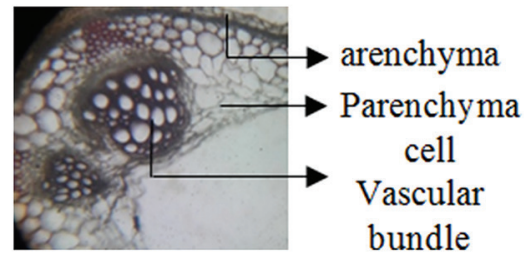

Fig. 5: Pitpapda (Stem part)

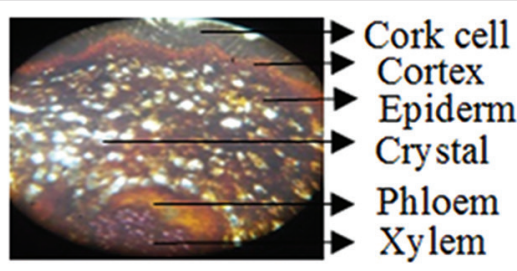

Fig. 3: Pasahan bheda (Root)

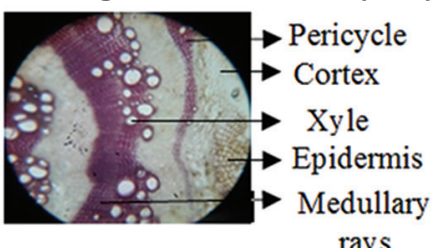

Fig. 6: Kaas (Root)

Table 3: Microscopical characters of TK churna

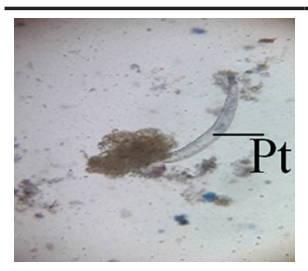

Fig. 7: Pitted tracheid

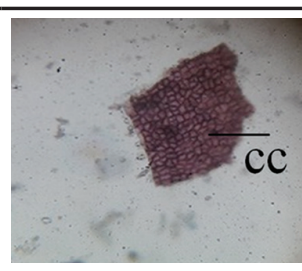

Fig. 8: Cork cell

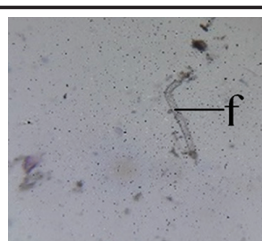

Fig. 9: Fiber

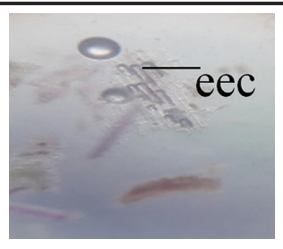

Fig. 10: Elongated cubical cell

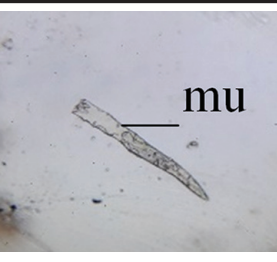

Fig. 11: Multicellular uniseriate

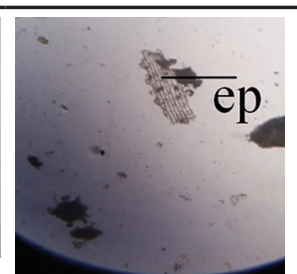

Fig. 12: Epidermal cell

Table 4: Master formula of formulations

\begin{tabular}{|c|c|c|c|c|c|c|c|c|c|}
\hline \multirow[t]{3}{*}{ Formulations } & \multicolumn{9}{|c|}{ Quantity } \\
\hline & \multicolumn{8}{|c|}{ Ingredients (g) } & \multirow[t]{2}{*}{ Other excipients (\%) } \\
\hline & G & A & D & $\mathbf{J}$ & $\mathbf{P}$ & $\mathbf{H}$ & $\mathbf{P a}$ & $\mathbf{K}$ & \\
\hline TK & 62.5 & 62.5 & 62.5 & 62.5 & 62.5 & 62.5 & 62.5 & 62.5 & Nil \\
\hline TKS & 62.5 & 62.5 & 62.5 & 62.5 & 62.5 & 62.5 & 62.5 & 62.5 & Preservative (50 S, $0.01 \mathrm{CA}, 0.2 \mathrm{PP}$ and MP) (\% w/v) \\
\hline TKGV & 20 & 20 & 20 & 20 & 20 & 20 & 20 & 20 & Gum acacia- $4(\% \mathrm{w} / \mathrm{w})$ \\
\hline TT & 25 & 25 & 25 & 25 & 25 & 25 & 25 & 25 & Ethanol- $15(\% \mathrm{v} / \mathrm{v})$ \\
\hline
\end{tabular}

TK: Trikantakadi kvatha, TKS: Trikantakadi kvatha syrup, TKGV: Trikantakadi kvatha Ghana vati, TT: Trikantakadi tincture, G: Gokshura, A: Amaltaas, D: Durbhamoola J: Javasa, P: Pashanbheda, H: Harar, Pa: Parpata, K: Kaasmoola, S: Sugar, CA: Citric acid, PP: Propylparaben, MP: Methylparaben 
$6.4 \mathrm{~g}$ of gum acacia as binding agent and were compressed into tablet form [10].

TT

$850 \mathrm{ml}$ (water) and $150 \mathrm{ml}$ (ethanol) were taken together, and then, $200 \mathrm{~g}$ of TK Dravya added. The entire wort was kept for extraction for the time period of 14 days (Table 4) [11].
Characterization of formulation

Determination of organoleptic characteristics such as color, odor, taste, and state of prepared formulations was carried out (Table 5 - Figs. 13-16). All the physicochemical parameters of prepared formulations were carried out, and results of the experiment are asserted in Tables 6 and 7 [12-14]. Phytochemical analysis and thin-layer chromatography (TLC) of formulation were performed

Table 5: Organoleptic characters of formulations

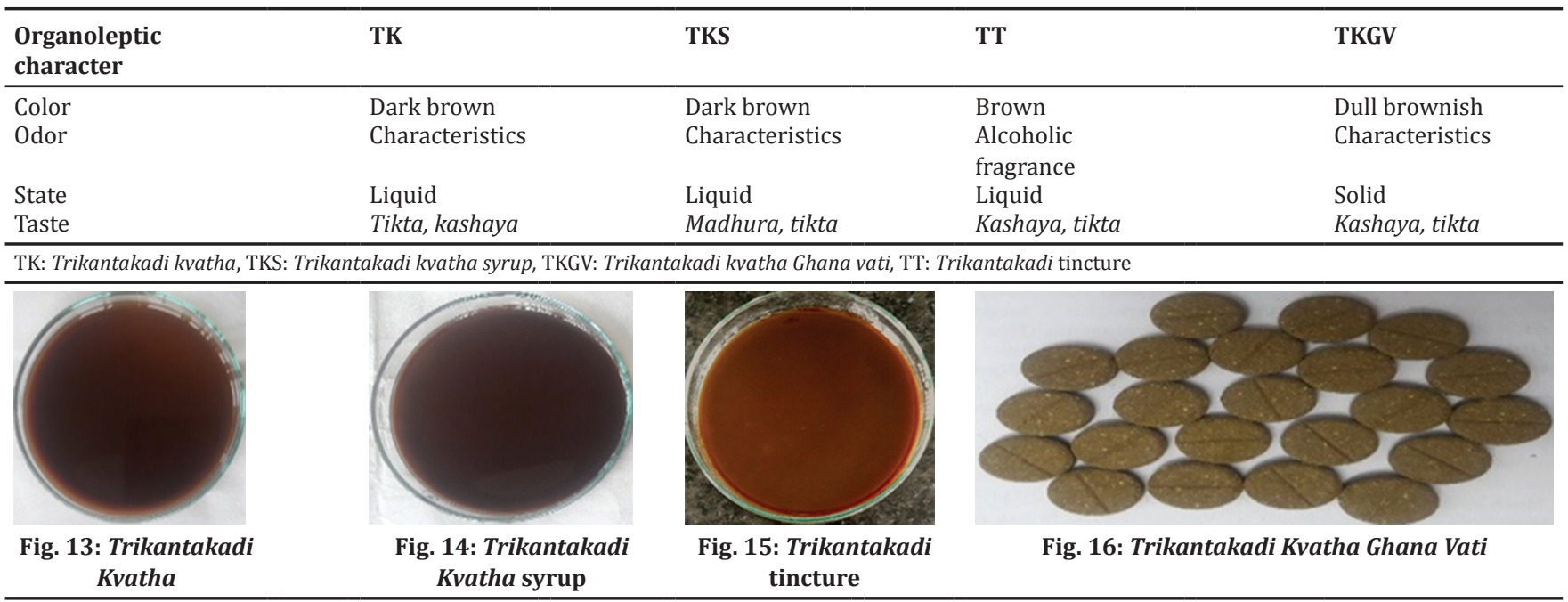

Table 6: Physicochemical parameters of formulations

\begin{tabular}{|c|c|c|c|c|c|c|c|c|c|c|c|c|}
\hline \multirow[t]{3}{*}{ Parameters } & \multicolumn{12}{|l|}{ Batch } \\
\hline & \multicolumn{4}{|c|}{ Kvatha } & \multicolumn{4}{|l|}{ Syrup } & \multicolumn{4}{|c|}{ Tincture } \\
\hline & K1 & K2 & K3 & Avg. & S1 & S2 & S3 & Avg. & T1 & $\mathbf{T 2}$ & T3 & Avg. \\
\hline Total ash $(\% \mathrm{w} / \mathrm{w})$ & 1.5 & 1 & 1 & 1.166 & 1.82 & 1.78 & 1.87 & 1.82 & 1.5 & 1.2 & 1.2 & 1.3 \\
\hline Acid insoluble ash(\%w/w) & 0.3 & 0.4 & 0.5 & 0.4 & 0.1 & 0.1 & 0.2 & 0.13 & 0.5 & 0.5 & 0.5 & 0.5 \\
\hline Total solid content ( $\% \mathrm{w} / \mathrm{v})$ & 19.32 & 19.31 & 19.33 & 19.32 & 41.82 & 43.58 & 41.98 & 42.47 & 3.5 & 3 & 3.2 & 3.24 \\
\hline pH meter & 4.77 & 4.77 & 4.78 & 4.77 & 4.58 & 4.59 & 4.59 & 4.58 & 4.76 & 4.75 & 4.76 & 4.756 \\
\hline Viscosity (millipoise) & 1.379 & 1.350 & 1.379 & 1.369 & 5.65 & 5.68 & 5.65 & 5.65 & 1.39 & 1.43 & 1.39 & 1.40 \\
\hline $\begin{array}{l}\text { Refractive index at room } \\
\text { temperature }\end{array}$ & 1.351 & 1.352 & 1.352 & 1.351 & 1.346 & 1.347 & 1.347 & 1.346 & 1.35 & 1.35 & 1.34 & 1.34 \\
\hline Alcohol content & NA & NA & NA & NA & NA & NA & NA & NA & 3 & 3 & 3 & 3 \\
\hline Total acidity (\%v/v) & NA & NA & NA & NA & 0.047 & 0.048 & 0.047 & 0.047 & 0.029 & 0.027 & 0.029 & 0.084 \\
\hline $\begin{array}{l}\text { Total sugar }(\% \mathrm{v} / \mathrm{v}) \text { titrimetric } \\
\text { method }\end{array}$ & NA & NA & NA & NA & 8.46 & 8.45 & 8.45 & 8.45 & 10.6 & 10.5 & 10.4 & 10.5 \\
\hline $\begin{array}{l}\text { Reducing sugar }(\% \mathrm{v} / \mathrm{v}) \\
\text { titrimetric method }\end{array}$ & NA & NA & NA & NA & 2.77 & 2.88 & 3.15 & 2.9 & 2.77 & 3.01 & 2.78 & 2.85 \\
\hline $\begin{array}{l}\text { Non- reducing sugar }(\% \mathrm{v} / \mathrm{v}) \\
\text { titrimetric method }\end{array}$ & NA & NA & NA & NA & 5.40 & 5.52 & 5.80 & 5.57 & 7.43 & 7.88 & 7.88 & 7.73 \\
\hline
\end{tabular}

Table 7: Test for tablets

\begin{tabular}{lc}
\hline Parameters & Observed result \\
\hline Appearance & Dull-brownish color, smooth surface \\
Shape & Round \\
Hardness & $4 \mathrm{~kg}^{\mathrm{inch}} \mathrm{inc}^{2}$ \\
Thickness and diameter & $4 \mathrm{~mm}, 10.5 \mathrm{~mm}$ \\
Friability & $1.001 \% \mathrm{w} / \mathrm{w}$ \\
Weight variation & $1.8 \% \mathrm{w} / \mathrm{w}$ \\
Disintegration time & $14 \mathrm{minutes}$ \\
\hline
\end{tabular}


Table 8: Phytochemical identification of formulations

\begin{tabular}{|c|c|c|c|c|c|}
\hline Chemical constituents & Test & TK & TKS & TT & TKGV \\
\hline \multirow[t]{3}{*}{ Alkaloid } & Mayer's reagent & + & + & + & + \\
\hline & Dragendorff's reagent & + & + & + & + \\
\hline & Wagner's reagent & + & + & + & + \\
\hline \multirow[t]{2}{*}{ Tannin } & Ferric chloride test & + & + & + & + \\
\hline & Lead acetate test & + & + & + & + \\
\hline Anthraquinone glycoside & Borntrager's test & + & + & + & + \\
\hline \multirow[t]{2}{*}{ Sterol/Steroids } & Legal test & + & + & + & + \\
\hline & Salkowski test & + & + & + & + \\
\hline Flavonoids & Shinoda test & + & + & + & + \\
\hline \multirow[t]{2}{*}{ Test for terpenoids } & Libermann-burchard's test & + & + & + & + \\
\hline & Salkowski test & - & - & - & - \\
\hline \multirow[t]{2}{*}{ Reducing sugar } & Benedict test & NA & - & + & NA \\
\hline & Fehling test & NA & - & + & NA \\
\hline Non reducing sugar & Benedict test & NA & + & - & NA \\
\hline
\end{tabular}

+: Present, -: Absent, NA: Not applicable. TK: Trikantakadi kvatha, TKS: Trikantakadi kvatha syrup, TKGV: Trikantakadi kvatha Ghana vati, TT: Trikantakadi tincture

Table 9: Thin-layer chromatography profile of TK, TKGV, TKS, and TT

\begin{tabular}{|c|c|c|c|c|}
\hline $\begin{array}{l}\text { Mobile phase- Ethyl } \\
\text { acetate: Methanol: Ethanol: } \\
\text { Water (81:11:4:8) } \\
\text { Stationary phase - silica gel G } \\
\text { Extract used for spotting: } \\
\text { Methanolic extract }\end{array}$ & $\begin{aligned} & \rightarrow \\
& \rightarrow \\
& \rightarrow \\
& \rightarrow \\
& \rightarrow\end{aligned}$ & $\rightarrow$ & 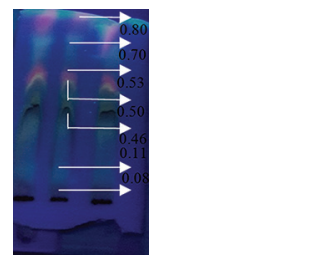 & $\stackrel{\rightarrow}{\rightarrow} \quad \rightarrow$ \\
\hline & $\begin{array}{l}\quad \begin{array}{l}\text { Fig. 17: Trikantakadi } \\
\quad \text { Kvatha }\end{array} \\
\text { Observed Rf value under } \\
365 \mathrm{~nm}: 0.08,0.11,0.28, \\
0.34,0.44,0.50,0.53,0.70, \\
0.80\end{array}$ & $\begin{array}{l}\text { Fig. 18: Trikantakadi } \\
\text { Kvatha Ghana Vati } \\
\text { Observed Rf value under } \\
365 \mathrm{~nm}: 0.11,0.28,0.44 \text {, } \\
0.53,0.70,0.80\end{array}$ & $\begin{array}{c}\text { Fig. 19: Trikantakadi } \\
\quad \text { Kvatha syrup } \\
\text { Observed Rf value under } \\
365 \mathrm{~nm}: 0.08,0.11,0.46 \text {, } \\
0.50,0.53,0.70,0.80\end{array}$ & $\begin{array}{l}\text { Fig. 20: Trikantakadi } \\
\quad \text { tincture } \\
\text { Observed Rf value under } \\
365 \mathrm{~nm}: 0.34,0.53,0.70 \text {, } \\
0.80\end{array}$ \\
\hline
\end{tabular}

TK: Trikantakadi kvatha, TKS: Trikantakadi kvatha syrup, TKGV: Trikantakadi kvatha Ghana vati, TT: Trikantakadi tincture

(Tables 8 and 9 - Figs. 17-20) [9,15]. Stability study of TKS, TT, and TKGV at accelerated temperature conditions was performed (Table 10) [16].

\section{OBSERVATION AND RESULT}

Pharmacognostic, physicochemical, phytochemical parameters of all the raw ingredients and formulations were studied, it showed that all the chemical compounds that were present in the Kwath (TK) were also present in other prepared dosage form. Stability studies of various prepared dosage forms of trikantakadi kwath was done for the time period of three days and during the stability studies of the various physicochemical, phytochemical and Thin Layer Chromatographic studies were done within the specific interval of time. Stability studies showed no significant variation when compared the observed results of accelerated temperature conditions data with the previous data.

\section{Method for stability study}

Stability study was performed by keeping the prepared samples at accelerated temperature conditions. Nine samples of each prepared dosage forms were taken and kept it at accelerated temperature of $4^{\circ} \mathrm{C}$ and room temperature of $47^{\circ} \mathrm{C}$. The samples were tested for the physicochemical properties like color, odor, $\mathrm{pH}$, specific gravity, friability, weight variation, hardness, etc. at the interval of $24 \mathrm{hrs,} 48 \mathrm{hrs,} \mathrm{and} 72$ hrs to observing the changes in physicochemical properties (Table 10).

\section{DISCUSSION}

TK was prepared by classical method mentioned in literature and converted into TKS, TKGV, and TT. Precaution should be taken during the processing of formulations. TT placed at dark place in airtight container. Physicochemical (Tables 6 and 7) and phytochemical investigation (Table 8) not showed remarkable variations with various prepared dosage forms. The $\mathrm{R}_{\mathrm{f}}$ range (Table 9 - Figs. 17-20) observed between the 0.08 and 0.80 follow the standard value when compared with the reference of plant drug used for the preparation of dosage form. Result of accelerated stability study (Table 10) of TKS, TT, and TKGV was not showed any remarkable changes in physicochemical properties when compared with the previous data (Tables 6-8). Hence, these results may make some improvement in stability and shelf life degradation studies.

\section{CONCLUSION}

TK is a polyherbal formulation used for treating the Ashmari, Mutraghat, and Mutrakricha. Using stander TK as base, various conventional dosage forms can be prepared. Physicochemical, phytochemical parameters, and TLC showed all chemical compounds that present in the Kvatha are also present in other prepared dosage form. Stability studies showed no remarkable variation with physicochemical properties when comparing the observed values, which done in the interval of $24 \mathrm{hrs}, 48 \mathrm{hrs}$, and 
Table 10: Stability studies through Physicochemical parameters of TKS, TT, and TKGV

\begin{tabular}{|c|c|c|c|c|c|c|c|c|c|c|c|c|c|c|c|}
\hline \multirow{2}{*}{$\begin{array}{l}\text { Sample } \\
\text { code }\end{array}$} & \multirow{2}{*}{$\begin{array}{l}\text { Time } \\
\text { duration } \\
\text { (in hour) }\end{array}$} & \multirow[t]{2}{*}{ Temperature $\left({ }^{\circ} \mathrm{C}\right)$} & \multicolumn{13}{|c|}{ Physicochemical parameters } \\
\hline & & & $\mathrm{C}$ & 0 & Ts & pH & Sp. & $\mathbf{R}$ & $\mathbf{V}$ & $\mathbf{T u}$ & $\mathbf{H}$ & Ha & $\mathbf{F}$ & $\mathbf{W}$ & D.T. \\
\hline SA1 & $24 \mathrm{hrs}$ & $4^{\circ} \mathrm{C}$ & $\mathrm{NC}$ & NC & $\mathrm{NC}$ & 4.58 & 1.18 & 1.346 & 5.6 & $\mathrm{X}$ & $\mathrm{Y}$ & NA & NA & NA & NA \\
\hline TA1 & & & $\mathrm{NC}$ & $\mathrm{NC}$ & $\mathrm{NC}$ & 4.76 & 1.00 & 1.342 & 1.3 & $\mathrm{X}$ & Y & NA & NA & NA & NA \\
\hline TaA1 & & & $\mathrm{NC}$ & $\mathrm{NC}$ & $\mathrm{NC}$ & NA & NA & NA & NA & NA & NA & 4 & 1.001 & 1.8 & 14 \\
\hline SA2 & & Room temperature & $\mathrm{NC}$ & $\mathrm{NC}$ & $\mathrm{NC}$ & 4.58 & 1.18 & 1.346 & 5.6 & $\mathrm{X}$ & $\mathrm{Y}$ & NA & NA & NA & NA \\
\hline TaA2 & & & $\mathrm{NC}$ & $\mathrm{NC}$ & $\mathrm{NC}$ & NA & NA & NA & NA & NA & NA & 4 & 1.001 & 1.8 & 14 \\
\hline SA3 & & $47^{\circ} \mathrm{C}$ & $\mathrm{NC}$ & $\mathrm{NC}$ & $\mathrm{NC}$ & 4.58 & 1.17 & 1.346 & 5.6 & $\mathrm{X}$ & $\mathrm{Y}$ & NA & NA & NA & NA \\
\hline TA3 & & & $\mathrm{NC}$ & $\mathrm{NC}$ & $\mathrm{NC}$ & 4.76 & 1.00 & 1.342 & 1.3 & $X$ & $\mathrm{Y}$ & NA & NA & NA & NA \\
\hline TaA3 & & & $\mathrm{NC}$ & $\mathrm{NC}$ & $\mathrm{NC}$ & NA & NA & NA & NA & NA & NA & 4 & 1.001 & 1.8 & 14 \\
\hline SB1 & 48 hrs & $4^{\circ} \mathrm{C}$ & $\mathrm{NC}$ & $\mathrm{NC}$ & $\mathrm{NC}$ & 4.58 & 1.17 & 1.346 & 5.6 & $X$ & Y & NA & NA & NA & NA \\
\hline TB1 & & & $\mathrm{NC}$ & $\mathrm{NC}$ & $\mathrm{NC}$ & 4.76 & 1.00 & 1.342 & 1.3 & $X$ & $\mathrm{Y}$ & NA & NA & NA & NA \\
\hline SB2 & & Room temperature & $\mathrm{NC}$ & $\mathrm{NC}$ & $\mathrm{NC}$ & 4.58 & 1.17 & 1.346 & 5.6 & $\mathrm{X}$ & Y & NA & NA & NA & NA \\
\hline TB2 & & & $\mathrm{NC}$ & $\mathrm{NC}$ & $\mathrm{NC}$ & 4.76 & 1.00 & 1.342 & 1.3 & $\mathrm{X}$ & $\mathrm{Y}$ & NA & NA & NA & NA \\
\hline TaB2 & & & $\mathrm{NC}$ & $\mathrm{NC}$ & NC & NA & NA & NA & NA & NA & NA & 4 & 1.001 & 1.8 & 14 \\
\hline SB3 & & $47^{\circ} \mathrm{C}$ & $\mathrm{NC}$ & $\mathrm{NC}$ & NC & 4.58 & 1.18 & 1.346 & 5.6 & $\mathrm{X}$ & Y & NA & NA & NA & NA \\
\hline TB3 & & & NC & $\mathrm{NC}$ & NC & 4.76 & 1.00 & 1.342 & 1.3 & $X$ & Y & NA & NA & NA & NA \\
\hline TaB3 & & & $\mathrm{NC}$ & $\mathrm{NC}$ & $\mathrm{NC}$ & NA & NA & NA & NA & NA & NA & 4 & 1.001 & 1.8 & 14 \\
\hline SC1 & $72 \mathrm{hrs}$ & $4^{\circ} \mathrm{C}$ & $\mathrm{NC}$ & $\mathrm{NC}$ & $\mathrm{NC}$ & 4.58 & 1.17 & 1.346 & 5.6 & $\mathrm{X}$ & $\mathrm{Y}$ & NA & NA & NA & NA \\
\hline TC1 & & & $\mathrm{NC}$ & $\mathrm{NC}$ & $\mathrm{NC}$ & 4.76 & 1.00 & 1.342 & 1.3 & $X$ & $\mathrm{Y}$ & NA & NA & NA & NA \\
\hline $\mathrm{TaC} 1$ & & & $\mathrm{NC}$ & $\mathrm{NC}$ & $\mathrm{NC}$ & NA & NA & NA & NA & NA & NA & 4 & 1.001 & 1.8 & 14 \\
\hline $\mathrm{SC} 2$ & & Room temperature & $\mathrm{NC}$ & $\mathrm{NC}$ & $\mathrm{NC}$ & 4.58 & 1.17 & 1.346 & 5.6 & $\mathrm{X}$ & $\mathrm{Y}$ & NA & NA & NA & NA \\
\hline TC2 & & & $\mathrm{NC}$ & $\mathrm{NC}$ & $\mathrm{NC}$ & 4.76 & 1.00 & 1.342 & 1.3 & $X$ & $\mathrm{Y}$ & NA & NA & NA & NA \\
\hline $\mathrm{TaC} 2$ & & & $\mathrm{NC}$ & $\mathrm{NC}$ & $\mathrm{NC}$ & NA & NA & NA & NA & NA & NA & 4 & 1.001 & 1.8 & 14 \\
\hline $\mathrm{TaC} 3$ & & & $\mathrm{NC}$ & $\mathrm{NC}$ & $\mathrm{NC}$ & NA & NA & NA & NA & NA & NA & 4 & 1.001 & 1.8 & 14 \\
\hline
\end{tabular}

C: Color, O: Odor, Ts: Taste, Sp.: Specific gravity at room temperature (g/ml), R: Refractive index at room temperature, V: Viscosity (millipoise), Tu: Turbidity, H: Homogeneity, Ha: Hardness (Kg/inch square), F: Friability (\%w/w), W: Weight variation (\%w/w), D.T.: Disintegration time (minute), S: Syrup, T: Tincture, Ta: Tablet, NC: No change, X: No, Y: Yes, NA: Not applicable. TKS: Trikantakadi kvatha syrup, TKGV: Trikantakadi kvatha Ghana vati, TT: Trikantakadi tincture

72 hrs. Hence, shelf life and all other related issue of Kvatha may be solve by converting Kvatha into most convenient dosage formed as per requirement.

\section{ACKNOWLEDGMENT}

The authors are grateful to Lovely Professional University, Phagwara, for providing the necessary infrastructure and resources to carry out this research project.

\section{REFERENCES}

1. Tripathi B, editor. Sharanagadhara Samhita with "Dipika Hindi Commentary". Madhyam Khand. Ch. 9. Varanasi: Chaukhamba Subharti Prakashan; 2008.

2. Shastri S. Agnivesha Charaka Samhita, Sutra Sthana 4/6. Varanasi: Chaukhamba Bharti Academy; 2006. p. 71.

3. Sushruta. Sushruta Samhita, Chikitsa Sthana 31/8. Varanasi: Chaukhambha Sanskrit Sansthan; 2005.

4. Tripathi RD. Astanga Samgraha, Sutra Sthana 178/103. Varanasi: Chaukhamba Sanskrit Pratisthan; 2003.

5. Pandit Sarangadhara. Sarangadhara Samhita, Madhyam Khand 2/3. Varanasi: Chaukhamba Surbharti Prakshana; 2010. p. 133-4.

6. Jha CB. Yogatarangini 18/20. Varanasi: Chaukhamba Vidyabhavan; 2003. p. 76.

7. Narayan SR. Ayurveda Sar Samghara, Reprint. $11^{\text {th }}$ ed. New Delhi:
Vaidyanatha Ayurved Bhavan; 2011.p. 707

8. Krishna G. Rasatantrasaar Va Sidhapryoga Samghra, ${ }^{\text {st }}$ Part. Ajmair: Krishna Gopal Ayurved Bhavan; 2005. p. 704.

9. Gupta AK, Tandan N, Sharma M: Quality Standard of Indian Medicinal Plants. Vol. I, II, IV, VII, VIII. New Delhi: Indian Council of Medicinal Research; 2003, 2005, 2006, 2008, 2010.

10. Bharat DK, Patgiri BJ, Prajapati PK. Standard manufacturing procedure for syrup and tablet forms of Jwarahara Dashemani. Int Q J Res Ayurveda 2010;31(2):255-9.

11. Lean L, Liebermon HA, Kaning JL. The Theory and Practice of Industrial Pharmacy. $3^{\text {rd }}$ ed. Bombay: Varghese Publishing House; 1987.

12. Anonymous. Protocol for Testing Ayurveda, Siddha and Unani medicines. Ghaziabad: Government of India, Ministry of Health and Family Welfare, Department of AYUSH, PLIM; 2006.

13. Anonymous. The Ayurvedic Pharmacopoeia of India Part-I. Appendix-2,3,5. $1^{\text {st }}$ ed., Vol. VI. New Delhi: Government of India, Ministry of Health and Family Welfare Department of Ayush; 2008.

14. Khar RK, Vyas SP, Ahmad FJ, Jain GK, Lachman L, Lieberman HA. The Theory and Practice of Industrial Pharmacy. $4^{\text {th }}$ ed. Philippines: CBS Publishers \& Distributors Pvt Ltd.; 2014.

15. Kokate CK. Practical Pharmacognosy. New Delhi: Vallabha Prakashan; 1994.

16. Kumar SP, Prasan ND. Development and evaluation of polyherbal syrup from some herbs used as expectorant. World J Pharm Pharm Sci 2013;2(5):3848-53. 